\title{
Das tödliche Trio bei jungen Erwachsenen
}

\author{
Die Kombination von milder Hypertonie, Alkoholkonsum und Rauchen erhöht schon im \\ frühen Lebensalter das Risiko für schwere kardiovaskuläre und renale Komplikationen.
}

In eine prospektive Kohortenstudie wurden 1.204 Patienten im Alter zwischen 18 und 45 Jahren (im Mittel 33) eingeschlossen. Sie alle hatten eine unbehandelte Hypertonie im Stadium I mit Blutdruckwerten von $140-159 \mathrm{mmHg}$ systolisch oder 90-99 $\mathrm{mmHg}$ diastolisch (im Mittel 145/93) ohne nennenswerte andere Risikofaktoren. Festgelegt wurden jeweils vier Abstufungen für den Konsum von Alkohol (0, < 50, 50-100 und $>100 \mathrm{~g} / \mathrm{d})$ und Zigaretten (0, 1-5, 6-10 und $>10$ pro Tag).

Innerhalb von 12,6 Jahren ereigneten sich 74 schwere tödliche und nicht-tödliche kardiovaskuläre (vor allem korona- re) und renale Komplikationen. Dabei stieg das Risiko über alle Kategorien des Alkohol- und Zigarettenkonsums um das 2- bis 4-Fache an.

Besonders risikoträchtig war die Kombination beider Faktoren, weil sich eine synergistische Wirkung ergab. Starke Raucher mit Alkoholkonsum hatten ein 7-mal höheres Risiko als nicht rauchende Abstinenzler. Diese Korrelationen veränderten sich bei Berücksichtigung anderer gesicherter Risikofaktoren nur wenig.

- Palatini P et al. Alcohol intake more than doubles the risk of early cardiovascular events in young hypertensive smokers. Am J Med. 2017;130:967-74

\section{KOMMENTAR}

Die Fachgesellschaften empfehlen, es bei Personen mit milder Hypertonie und niedrigem kardiovaskulären Risiko zunächst über 6-12 Monate mit Allgemeinmaßnahmen und ohne Antihypertensiva zu versuchen. Grundlage sind Daten älterer Patienten. Nun zeigt sich aber, dass junge Hypertoniker mit Nikotinoder Alkoholkonsum ein deutlich erhöhtes Gefäßrisiko haben. Sie sollten deshalb auf Alkohol und Zigaretten verzichten und sofort antihypertensiv behandelt werden. Eine kardioprotektive Wirkung des Alkohols ergab sich nicht.

Prof. Dr. med. H. Holzgreve

\section{Nach 13 Stunden am Boden ist der Arm fast tot}
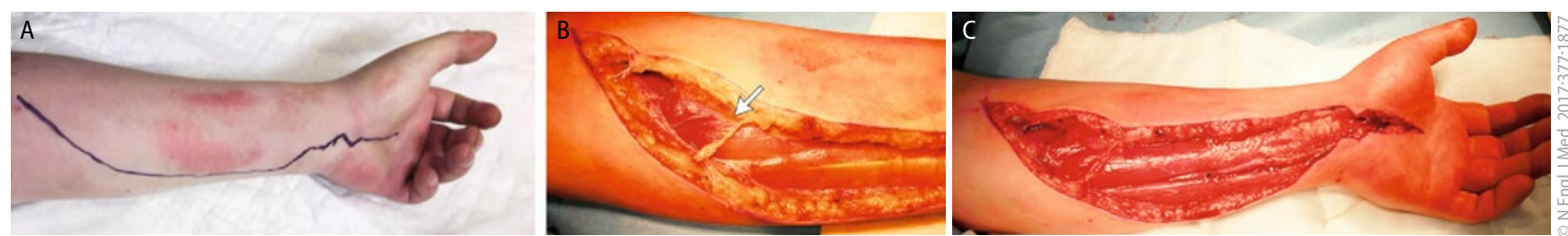

A: Schwellung der linken Hand. B: Klare Linie zwischen durchbluteten und ischämischen Muskelpartien (Pfeil). C: Wiederhergestellte Durchblutung nach chirurgischer Dekompression.

Im Drogenrausch lag ein 26-jähriger Mann 13 Stunden lang am Boden. Danach musste er wegen einer schmerzhaften Schwellung der linken Hand (Abb. A) mit bläulich-livider Verfärbung der Finger in die Nothilfe gebracht werden. An Hand und Unterarm bestand ein Ödem. Die aktive und passive Handbeweglichkeit war schmerzhaft eingeschränkt. Der Puls der A. ulnaris war tastbar, nicht aber der Radialispuls. Auch die kapilläre Füllung war verzögert. Zusätzlich bestand eine Sensibilitätsstörung im Verbreitungsgebiet aller drei Unterarmnerven. Im Labor fand sich eine CK-Konzentration von $21.500 \mathrm{U} / \mathrm{I}$ als Zeichen für einen Muskelzelluntergang.

Unter der Diagnose eines Kompartment-Syndroms erfolgte in Blutleere eine chirurgische Dekompression, wobei sich eine klare Demarkierung zwischen durchbluteten und ischämischen, blassen und nichtkontraktilen Muskelanteilen abzeichnete (Abb. B). Nach Lockerung des Tourniquets konnte die Durchblutung wiederhergestellt werden (Abb. C).
Kompartment-Syndrome entstehen nach stumpfen Verletzungen oder langer Immobilisierung, wenn ein Hämatom oder ein Ödem den in der Faszie "gefangenen" Muskel von der Durchblutung abschnürt, sodass es zur Muskelnekrose und Nervenschädigung kommt. Eine rasche chirurgische Dekompression durch Spaltung der Faszie ist unbedingt erforderlich, um die Extremität zu retten. Bei einem verspäteten Eingriff wie im vorliegenden Fall bleiben in der Regel Schäden an Nerven und Muskulatur zurück.

48 Stunden nach dem ersten Eingriff wurde eine weitere Operation durchgeführt und die Wunde mit Spalthaut plastisch gedeckt. Der Patient nahm die empfohlene Ergotherapie nicht in Anspruch und behielt eine Steifheit der Hand und Parästhesien.

Wenigen Suchtpatienten ist klar, wie gefährlich es ist, intoxikiert völlig immobil in ungünstiger Lage auf dem Boden zu liegen. -

Prof. Dr. med. H. S. FüeßI

- Egan AF, Cahill KC. Compartment syndrome. N Eng/ J Med. 2017;377:1877 